\title{
Evaluación de la resistencia a insecticidas de una cepa de Aedes aegypti de El Salvador
}

\author{
Juan A. Bisset Lazcano, ${ }^{1}$ María M. Rodríguez, ${ }^{1}$ \\ José L. San Martín, ${ }^{2}$ José E. Romero y Romeo Montoya ${ }^{3}$
}

Forma de citar

Bisset Lazcano JA, Rodríguez MM, San Martín JL, Romero JE, Montoya R. Evaluación de la resistencia a insecticidas de una cepa de Aedes aegypti de El Salvador. Rev Panam Salud Publica. 2009;26(3): $229-34$.

RESUMEN Objetivos. Evaluar el nivel de susceptibilidad a insecticidas de una cepa de Aedes aegypti procedente de El Salvador y describir los posibles mecanismos de resistencia al temefós.

Métodos. Se evaluó una cepa de A. aegypti procedente del municipio de Soyapango, departamento de San Salvador, El Salvador. Mediante bioensayos se determinó la susceptibilidad de las larvas al insecticida organofosforado temefós y a tres piretroides (deltametrina, lambdacialotrina y cipermetrina) y de los adultos a un insecticida organofosforado (clorpirifós). Se determinó el factor de resistencia $\left(F R_{50}\right)$ con respecto a una cepa sensible de referencia (Rockefeller). Se estableció el mecanismo de resistencia al temefós mediante el empleo de sustancias sinergistas, ensayos bioquímicos de actividad enzimática y zimogramas en gel de poliacrilamida.

Resultados. Las larvas de la cepa estudiada mostraron una alta resistencia al temefós $\left(F R_{50}=24,16\right)$. De las enzimas analizadas, se encontró que solo la esterasa A4 estaba vinculada al mecanismo de resistencia al temefós. Los mosquitos adultos resultaron susceptibles a la lambdacialotrina y al clorpirifós y su resistencia a la deltametrina y la cipermetrina quedó en la categoría de verificación.

Conclusiones. La resistencia al temefós podría reducir la eficacia del control químico del mosquito A. aegypti en la zona estudiada de El Salvador. Los insecticidas clorpirifós, lambdacialotrina y cipermetrina son buenos candidatos alternativos a utilizar en las nuevas intervenciones de control de este vector.

Palabras clave Aedes; temefós; resistencia a los insecticidas; control vectorial; El Salvador.

Durante el año 2006, el dengue fue una de las enfermedades que más afectó a la población de El Salvador, especialmente en la región centrooccidental del país y a

\footnotetext{
1 Instituto de Medicina Tropical Pedro Kourí, Ciudad de La Habana, Cuba. La correspondencia se debe dirigir a Juan A. Bisset Lazcano, Instituto de Medicina Tropical Pedro Kourí, CP 601, Marianao 13, Ciudad de La Habana, Cuba. Correo electrónico: bisset@ipk.sld.cu

2 Programa Regional de Dengue, Organización Panamericana de la Salud, Ciudad de Panamá, Panamá.

3 Departamento de Entomología, Ministerio de Salud, San Salvador, El Salvador.
}

la población infantil (1). En esto ha desempeñado un importante papel el alto nivel de infestación con el mosquito Aedes aegypti L., que continúa siendo el vector más importante en la transmisión de la fiebre amarilla y el dengue en la Región de las Américas.

Hasta ahora, la manera más exitosa de reducir la incidencia de dengue es el control del vector, en lo que el uso de insecticidas químicos es un componente importante por su eficacia en reducir las poblaciones de larvas y adultos (2). Los insecticidas más utilizados son los organofosforados (temefós para la elimina- ción de larvas durante el tratamiento focal y el fentión, el fenitrotión y el malatión para la eliminación de los mosquitos adultos) y los piretroides (deltametrina, lambdacialotrina, cipermetrina y ciflutrina). Estos últimos se introdujeron a partir de la década de 1990 en la mayoría de los países de América Latina para el control de los mosquitos adultos, especialmente durante las epidemias o cuando hay altos índices de infestación con A. aegypti. En El Salvador se ha utilizado el temefós para el control larval de los mosquitos vectores de enfermedades y en los últimos nueve años se han em- 
pleado la deltametrina y la permetrina para el control de los mosquitos adultos en todo el país (3).

El desarrollo de la resistencia del mosquito A. aegypti a los insecticidas constituye el principal problema que afecta a las estrategias de control y se debe a la selección de genes de resistencia en las poblaciones de esta especie (4). Algunos autores han mencionado que el mecanismo asociado con la resistencia de $A$. aegypti a los insecticidas organofosforados podría estar vinculado con la elevación de las esterasas (5-9).

La Organización Mundial de la Salud (OMS) ha sugerido algunas alternativas para el control larval de este vector, como el metopreno a dosis no superiores de $1 \mathrm{mg} / \mathrm{L}$, el piriproxifeno a 0,01 mg/L $\mathrm{y}$ cultivos de Bacillus thuringiensis israelensis (10).

Todo lo anterior reafirma la necesidad de conocer mejor los mecanismos de resistencia de los mosquitos vectores al temefós y otros insecticidas, a fin de poder trazar mejores estrategias dirigidas a elevar el control de las enfermedades transmitidas por estos insectos. El objetivo del presente trabajo fue evaluar el nivel de susceptibilidad a insecticidas de una cepa de A. aegypti procedente de El Salvador y describir los posibles mecanismos de resistencia al temefós.

\section{MATERIALES Y MÉTODOS}

\section{Cepas utilizadas para el trabajo}

Para el presente estudio experimental se utilizaron las siguientes cepas:

Rockefeller: cepa de referencia de $A$. aegypti susceptible a insecticidas, suministrada por los Centros para el Control y la Prevención de Enfermedades (CDC), San Juan, Puerto Rico.

El Salvador: cepa de campo de $A$. aegypti colectada en 2007 en Colonia Las Margaritas Segunda Etapa, Municipio de Soyapango, en el centro de la región metropolitana del departamento de San Salvador. Este municipio está ubicado en la zona central del país y tiene una extensión territorial de $29,7 \mathrm{~km}^{2}$. La ciudad cabecera, Soyapango, abarca casi la totalidad del municipio. Las larvas colectadas se colonizaron y se trabajó con la descendencia F1 obtenida en el insectario del Instituto de Medicina Tropical Pedro Kourí, en La Habana, Cuba.
SANTEM-F11: cepa de A. aegypti procedente de Santiago de Cuba, Cuba, colectada en 1997 y seleccionada por 11 generaciones hasta alcanzar una resistencia al temefós 191,21 veces superior que la cepa susceptible de referencia Rockefeller.

\section{Insecticidas utilizados}

Temefós: o,o-dimetil fosforotioato odiéster con 4,4' tiodifenol; con 93,3\% de pureza, suministrado por American Cyanamid Co. (Princeton, NJ, Estados Unidos de América). Se utilizó a una concentración de 1,0\% en acetona y se hicieron diluciones de 1:10 para las evaluaciones de las diferentes dosis.

Clorpirifós: o,o-dietil-o-(3,5,6-tricloro2-piridil) fosforotioato; con $94 \%$ de pureza, suministrado por Down Chemical Co. (Midland, MI, Estados Unidos). Se utilizó a una concentración de 1,0\% en aceite de oliva.

Deltametrina: (S)- $\alpha$-cyano-3-phenoxybenzyl (1R,3R)-3-(2,2-dibromovinyl)-2,2dimethylcyclopropane carboxylate; con 96,8\% de pureza, suministrado por Roussel Uclaf (Romainville, Francia). Se utilizó a una concentración de $0,1 \%$ en silicona.

Cipermetrina: (1RS)-cis,trans-3-(2,2diclorovinil)-2,2-dimetilciclopropano carboxilato de (RS)-ciano-3-Fenoxibencilo (IUPAC); con 90,5\% de pureza, suministrada por Chemotécnica S.A. (Buenos Aires, Argentina). Se utilizó a una concentración de $0,1 \%$ en silicona.

Lambdacialotrina: (S)- $\alpha$-cyano-3-phenoxybenzyl (Z)-(1R,3R)-3-(2-chloro-3,3,3trifluoroprop-1-enyl)-2,2-dimethylcyclopropanecarboxylate; con 97,8\% de pureza, suministrada por Syngenta S.A. (Estocolmo, Suecia). Se utilizó a una concentración de $0,1 \%$ en silicona.

\section{Determinación de la susceptibilidad al temefós en larvas}

La susceptibilidad a los insecticidas se caracterizó mediante la concentración letal en la que muere $50 \%\left(\mathrm{CL}_{50}\right)$ o $90 \%$ $\left(\mathrm{CL}_{90}\right)$ de la población tratada. La resistencia de las larvas de A. aegypti al temefós se determinó mediante los bioensayos de susceptibilidad propuestos por la OMS (4). Para ello se colocaron 20 larvas que se encontraban en el tercer estadio tardío o el cuarto estadio temprano en vasos que contenían temefós en concentraciones de 0,001,0,003,0,005, 0,008,
$0,01,0,03,0,05,0,08$ y $0,1 \mathrm{mg} / \mathrm{L}$, con cinco réplicas por cada concentración del insecticida. Una vez establecidas las concentraciones que ocasionaron una letalidad entre $2 \%$ y $98 \%$ se repitieron los ensayos tres veces en días diferentes.

La letalidad se determinó 24 horas después de aplicado el insecticida y los resultados se analizaron mediante el programa probit-logaritmo de Raymond (11), el cual permite contrastar gráficamente la letalidad observada contra el logaritmo de la dosis de insecticida empleada.

Se calcularon los factores de resistencia al temefós para la $\mathrm{CL}_{50}\left(\mathrm{FR}_{50}\right)$ y la $\mathrm{CL}_{90}\left(\mathrm{FR}_{90}\right)$ como la razón entre la CL correspondiente de la cepa evaluada y el valor de esa CL en la cepa susceptible de referencia. Se consideró que la resistencia de una cepa era alta si el valor de $\mathrm{FR}_{50}$ era mayor de 10, moderada si estaba entre 5 y 10 y susceptible si era menor de 5 . Los bioensayos se realizaron en el laboratorio a una temperatura de $25 \pm 2{ }^{\circ} \mathrm{C}$ y una humedad relativa de $75 \pm 2 \%$.

\section{Determinación de los mecanismos de resistencia al temefós}

Los mecanismos de resistencia al temefós se evaluaron mediante ensayos in vivo con sustancias sinergistas e in vitro mediante ensayos bioquímicos.

Ensayos con sinergistas. Para estos ensayos in vivo se emplearon tres sustancias sinergistas: fosfato de trifenilo, que es un inhibidor de las esterasas; butóxido de piperonilo, que funciona como inhibidor de la enzima monooxigenasa; y ácido etacrínico, que inhibe la acción de la enzima glutatión S-transferasa (GST). Para ello se expusieron larvas del cuarto estadio a dosis subletales de $2,5 \mathrm{mg} / \mathrm{L}$ de fosfato de trifenilo, $5 \mathrm{mg} / \mathrm{L}$ de butóxido de piperonilo y $5 \mathrm{mg} / \mathrm{L}$ de ácido etacrínico durante 4 horas, previo a la adición de las diferentes concentraciones de insecticida, y se calcularon la $\mathrm{CL}_{50}$ a las 24 horas de exposición —con el programa probit-logaritmo (11) - y el valor del factor de sinergia (FS); se consideró que había sinergia cuando FS $>5$.

Ensayos bioquímicos. Se analizó la actividad enzimática, como marcador de posibles mecanismos de resistencia a insecticidas, en larvas de la cepa de trabajo colectada en El Salvador y de la cepa sus- 
ceptible de referencia Rockefeller. Para cada ensayo se utilizaron 352 larvas de cada cepa y se realizaron tres réplicas de cada ensayo.

La actividad de las esterasas se determinó en larvas del tercer estadio tardío o cuarto estadio temprano, según el método adaptado para A. aegypti por Rodríguez y colaboradores (6). Para ello se homogeneizó una larva en $200 \mu \mathrm{L}$ de tampón de fosfato a 0,01 M (pH 7,5). En una placa de microtitulación de poliestireno se colocaron $20 \mu \mathrm{L}$ del homogeneizado, se añadieron $200 \mu \mathrm{L}$ del sustrato (acetato de $\beta$-naftilo a 0,7 mM) y se dejó transcurrir la reacción por 10 minutos. Después se añadieron $40 \mu \mathrm{L}$ del indicador Fast Blue B y se leyó la densidad óptica (DO) a $570 \mathrm{~nm}$ en un lector para placas de ELISA Labsystems iMS Reader MF (Helsinki, Finlandia). Se consideró que una DO por encima de 1,2 reflejaba la presencia de esterasas.

El análisis del patrón isoenzimático o zimograma de esterasas se realizó mediante electroforesis en gel de poliacrilamida al $10 \%$, realizada a 150 voltios durante 45 minutos. Las bandas de esterasas se revelaron con los sustratos inespecíficos de las esterasas ( $\beta$-naftilacetato y $\alpha$-naftilacetato) y el colorante Fast Blue RR.

La actividad de GST, expresada en $\mu \mathrm{mol} / \mathrm{min}$, se determinó por el método de Booth y colaboradores (12) adaptado para A. aegypti (6). Brevemente, a $20 \mu \mathrm{L}$ de cada homogeneizado de larva se añadieron $250 \mu \mathrm{L}$ de una mezcla de 1-cloro2,4 dinitrobenceno a $50 \mathrm{mM}$ y glutatión reducido a $20 \mathrm{mM}$. Se dejó transcurrir la reacción por 3 minutos y se leyó la DO a $340 \mathrm{~nm}$. Se consideró que una DO por encima de 0,7 reflejaba la presencia de GST.

La actividad de la acetilcolinesterasa en larvas, tanto la normal como la inhibida con propoxur, se determinó por el método de Hemingway y colaboradores (13) a partir del homogeneizado de larvas individuales en $50 \mu \mathrm{L}$ de tampón de fosfato con $1 \%$ de tritón X-100. En una placa de microtitulación de poliestireno se colocaron $20 \mu \mathrm{L}$ de ácido 5-5' ditiobis 2 nitrobenzoico a $0,07 \mathrm{M}(\mathrm{pH} 7,5)$ y $20 \mu \mathrm{L}$ de acetiltiocolina yodada a 0,06 M. Para la determinación de la acetilcolinesterasa inhibida se agregaron, además, $10 \mu \mathrm{L}$ de propoxur a 0,025 M. Se añadieron $20 \mu \mathrm{L}$ del homogeneizado de larvas. La reacción se dejó transcurrir durante 30 minutos y se leyó la DO a $405 \mathrm{~nm}$. La actividad de la acetilcolinesterasa se expresó como actividad enzimática residual, calculada mediante la razón de la actividad enzimática sin inhibidor entre la actividad enzimática con inhibidor, expresada en porciento. Se consideró que los valores por encima de $60 \%$ reflejaban la presencia de acetilcolinesterasa modificada.

Se estimó la frecuencia de la presencia de esterasas, GST y acetilcolinesterasa modificada a partir del número de larvas que resultaron susceptibles en cada ensayo, bajo el presupuesto de que la población se encontraba en equilibrio según el principio de Hardy-Weinberg (no estaba bajo la presión de la selección natural $\mathrm{u}$ otro factor y no se produjo ninguna mutación importante).

\section{Determinación de la susceptibilidad a insecticidas en adultos}

Los bioensayos para determinar la susceptibilidad a los insecticidas piretroides (lambdacialotrina, cipermetrina y deltametrina) y organofosforado (clorpirifós) estudiados se realizaron según las normas de la OMS para mosquitos adultos (14). Brevemente: las hembras se expusieron durante 1 hora a papeles impregnados con lambdacialotrina $(0,1 \%)$, cipermetrina $(0,1 \%)$, deltametrina $(0,1 \%)$ y clorpirifós $(1,0 \%)$, por separado, en cilindros plásticos de exposición provistos por la OMS. Se realizaron cuatro réplicas con 25 mosquitos cada una y el experimento se repitió tres veces, para un total de 300 mosquitos por insecticida. Como control se utilizaron papeles impregnados con silicona. Los resultados se analizaron según los criterios y categorías de resistencia a insecticidas de la OMS (susceptibilidad: entre $98 \%$ y $100 \%$ de letalidad; verificación de la resistencia: entre $80 \%$ y $97 \%$; y alta resistencia: menos de $80 \%$ de letalidad).

\section{RESULTADOS}

Según los resultados de los ensayos realizados, las larvas de la cepa de El Salvador mostraron una alta resistencia al temefós $\left(\mathrm{FR}_{50}=24,16\right)$. Sin embargo, la resistencia fue desigual en las diferentes muestras, lo que se reflejó en el bajo valor de la pendiente $(b=0,64)$ de la recta probit-log (cuadro 1). La cepa SANTEM-F11, utilizada como referencia por su elevada resistencia al temefós, presentó altos valores de $\mathrm{FR}_{50}(191,21)$ y $\mathrm{FR}_{90}(179,52)$.

Los resultados del empleo de sustancias sinergistas indicaron que solo las esterasas estaban involucradas en la resistencia al temefós (FS > 5) cuando se empleó fosfato de trifenilo y en menor medida con el butóxido de piperonilo $(\mathrm{FS}=3,97)$ y el ácido etacrínico (FS $=1,21)$ (cuadro 2). Esto indica que la resistencia de la cepa de El Salvador a este insecticida organofosforado no está relacionada con la monooxigenasa o la GST.

Los ensayos bioquímicos realizados confirmaron que solo las esterasas participaron en la resistencia al temefós. Como se observa en el cuadro 3, la frecuencia de estas enzimas en las larvas de El Salvador fue de $61,5 \%$. La frecuencia de la presencia de GST fue baja $(3,4 \%)$ y en ninguna larva se detectó la presencia de acetilcolinesterasa modificada.

En la electroforesis en gel de poliacrilamida se observó una banda coincidente con una ya clasificada como esterasa A4 en la cepa de referencia SANTEEM-F11, resistente al temefós (6). Esta banda no se encontró en la cepa susceptible de referencia Rockefeller (figura 1).

Los ensayos de resistencia a insecticidas piretroides (deltametrina, lambdacialotrina y cipermetrina) y organofosforado (clorpirifós) en mosquitos adultos demostraron que los ejemplares de la

CUADRO 1. Resistencia al temefós en larvas de Aedes aegypti, colectadas en El Salvador y de las cepas de referencia Rockefeller (susceptible) y SANTEM-F11 (resistente)

\begin{tabular}{llccccccc}
\hline \multicolumn{1}{c}{ Cepas } & $\mathrm{CL}_{50}$ & $\mathrm{IC} 95 \%$ & $\mathrm{CL}_{90}$ & $\mathrm{IC} 95 \%$ & $\mathrm{FR}_{50}$ & $\mathrm{FR}_{90}$ & $b$ & $\begin{array}{c}\text { Desviación } \\
\text { estándar }\end{array}$ \\
\hline El Salvador & 0,029 & $0,026-0,033$ & 0,082 & $0,071-0,990$ & 24,16 & 6,83 & 0,64 & 0,16 \\
Rockefeller & 0,0012 & $0,0009-0,0010$ & 0,012 & $0,007-0,030$ & - & - & 1,27 & 0,33 \\
SANTEM-F11 & 0,69 & $0,64-0,76$ & 1,49 & $1,29-1,85$ & 191,21 & 179,52 & 3,85 & 0,37 \\
\hline
\end{tabular}

Abreviaciones: $\mathrm{CL}_{50}$ : concentración letal en la que muere $50 \%$ de las larvas, en $\mathrm{mg} / \mathrm{L} ; \mathrm{CL}_{90}$ : concentración letal en la que muere $90 \%$ de las larvas, en $\mathrm{mg} / \mathrm{L}$; IC95\%: intervalo de confianza de $95 \%$; $\mathrm{FR}_{50}$ : factor de resistencia, razón entre las $\mathrm{CL}_{50}$ de la cepa a evaluar y de la cepa de referencia (Rockefeller); $\mathrm{FR}_{90}$ : factor de resistencia, razón entre las $\mathrm{CL}_{90}$ de la cepa a evaluar y de la cepa de referencia (Rockefeller); $b$ : pendiente de la recta probit-log que relaciona la letalidad con el logaritmo de la concentración de insecticida.

a $\mathrm{Se}$ evaluaron 1000 larvas. 
CUADRO 2. Resistencia al temefós en larvas de Aedes aegypti, colectadas en EL Salvador, tratadas previamente con sinergistas

\begin{tabular}{lccrrr}
\hline Sinergista empleado & $\mathrm{CL}_{50}$ & $\mathrm{IC} 95 \%$ & $\mathrm{FS}$ & $b$ & $\begin{array}{c}\text { Desviación } \\
\text { estándar }\end{array}$ \\
\hline Fosfato de trifenilo & 0,0029 & $0,0024-0,0034$ & 10,00 & 3,06 & 0,37 \\
Butóxido de piperonilo & 0,0073 & $0,0045-0,0098$ & 3,97 & 2,19 & 0,34 \\
Ácido etacrínico & 0,0240 & $0,0180-0,0270$ & 1,21 & 4,63 & 0,79 \\
\hline
\end{tabular}

Abreviaciones: $\mathrm{CL}_{50}$ : concentración letal en la que muere $50 \%$ de las larvas, en $\mathrm{mg} / \mathrm{L}$; IC95\%: intervalo de confianza de $95 \%$ FS: factor de sinergia, razón entre las $\mathrm{CL}_{50}$ obtenidas con temefós sin el sinergista y con temefós con el sinergista; $b$ : pendiente de la recta probit-log que relaciona la letalidad con el logaritmo de la concentración de insecticida.

CUADRO 3. Marcadores de mecanismos de resistencia en larvas de Aedes aegypti de una cepa procedente de El Salvador y de la cepa de referencia Rockefeller (susceptible)

\begin{tabular}{lcc}
\hline \multirow{1}{*}{ Marcador } & \multicolumn{2}{c}{$\begin{array}{c}\text { Larvas con el } \\
\text { marcador (\%) }\end{array}$} \\
\cline { 2 - 3 } & Rockefeller & El Salvador \\
\hline Esterasas & 0 & 61,5 \\
Glutation S-transferasa & 0 & 3,4 \\
$\begin{array}{l}\text { Acetilcolinesterasa } \\
\text { modificada }\end{array}$ & 0 & 0,0 \\
\hline
\end{tabular}

Se evaluaron 352 larvas por cada mecanismo de resistencia. Se presenta el valor promedio de tres réplicas de cada ensayo.

cepa de El Salvador se pueden clasificar, según los criterios de la OMS, en la categoría de "verificación" por su nivel de resistencia a la deltametrina y la cipermetrina, y como susceptibles a la lambdacialotrina y al clorpirifós (cuadro 4).

\section{DISCUSIÓN}

En la ciudad de Soyapango, cuarta ciudad más poblada de El Salvador, existen factores socioambientales que facilitan altas tasas de infestación con el mosquito vector del dengue, por lo que la tasa de transmisión de esta enfermedad es alta. El suministro irregular de agua que afecta a la mitad de sus colonias y comunidades - lo que los obliga a almacenar agua en recipientes y tanques-y el deficiente sistema de disposición de desechos sólidos elevan considerablemente la probabilidad de que surjan epidemias de dengue.

En El Salvador se ha utilizado el insecticida organofosforado temefós tanto para el control de los vectores de la malaria como del dengue (3), lo que puede explicar que en la localidad estudiada se haya detectado resistencia a este insecticida. Estos resultados, obtenidos en ensayos de laboratorio, subrayan la necesidad de conocer y monitorear la resistencia al temefós en este municipio y en el resto del país y de realizar pruebas de efectividad del producto comercial en condiciones de campo para evitar que la resistencia se convierta en un problema irreversible. Por su relación costo-efectividad, este insecticida continúa siendo la mejor alternativa para el control de las larvas de $A$. aegypti.

En 1999, la Fundación Nacional Brasileña para la Salud comenzó el primer Programa Nacional de Monitoreo de la resistencia a insecticidas y encontró resistencia al temefós en varios municipios de los estados de Río de Janeiro, Espíritu Santo, Sergipe y Alagoas $(15,16)$. Otros autores han demostrado también la tendencia al aumento de la resistencia al temefós en Brasil $(17,18)$ y otros países como Tailandia (19), por lo que se recomienda la aplicación de otros métodos de control alternativos para poder preservar la efectividad de este insecticida para la eliminación de las larvas de mosquitos vectores.

Las sustancias sinergistas son compuestos químicos que inhiben de manera específica algunas enzimas involucradas en el metabolismo de los insecticidas, con lo que potencian su acción. En este trabajo se demostró que con el empleo del fostato de trifenilo - un inhibidor específico de las esterasas- se logró reducir 10 veces la resistencia al temefós $(\mathrm{FS}=10,0)$, lo que confirma que la resistencia detectada a este insecticida estaba dada por el incremento de la actividad de las esterasas y no de las otras enzimas de acción metabólica (monooxigenasa y glutatión S-transferasa), cuyos valores de FS resultaron bajos.

La actividad de las enzimas metabólicas involucradas en la resistencia puede incrementarse cuantitativamente o puede alterarse su centro catalítico, con lo que mejoraría su acción específica sobre uno o más insecticidas. El incremento de esa actividad, detectado en este trabajo mediante ensayos bioquímicos, demostró una mayor actividad de las esterasas en gran parte de la muestra estudiada. Esto puede explicar la alta resistencia al temefós encontrada. El incremento en la actividad de las esterasas se ha asociado con la resistencia del mosquito $A$. aegypti al temefós en Venezuela (4), Trinidad y Tobago (20), Cuba $(9,21-22)$, Perú (23), Tailandia $(24)$ y Brasil $(17,25)$.

La presencia en el zimograma de una banda de esterasa en las muestras de El Salvador, coincidente con la encontrada en las larvas de la cepa resistente SANTEEM-F11 —clasificada como esterasa $\mathrm{A} 4$, por su movilidad relativa y su especificidad para reaccionar con el sus-

\begin{abstract}
FIGURA 1. Zimograma de esterasas en larvas de una cepa de Aedes aegypti procedente de El Salvador (carriles 1-6), la cepa resistente de referencia SANTEM-F11 (carril 8) y la cepa susceptible de referencia Rockefeller (carriles 9 y 10)
\end{abstract}

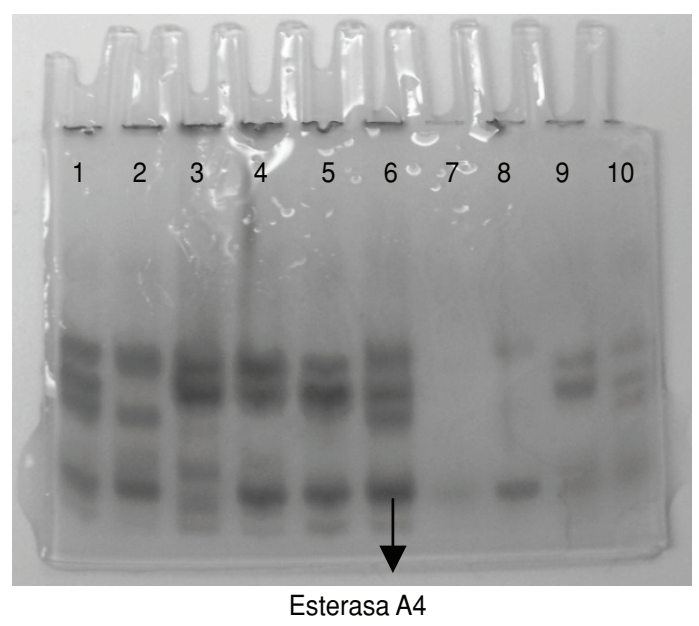


CUADRO 4. Resistencia a insecticidas en mosquitos adultos Aedes aegypti de una cepa procedente de El Salvador y de la cepa de referencia Rockefeller (susceptible)

\begin{tabular}{lccc}
\hline & & \multicolumn{2}{c}{ Letalidad (\%) ${ }^{\mathrm{a}}$} \\
\cline { 3 - 4 } Insecticidas & Concentración (\%) & El Salvador & Rockefeller \\
\hline Piretroides & & & \\
$\quad$ Deltametrina & 0,1 & 93,4 & 100,0 \\
$\quad$ Lambdacialotrina & 0,1 & 100,0 & 100,0 \\
$\quad$ Cipermetrina & 0,1 & 95,2 & 100,0 \\
Organofosforados & & 100,0 & 100,0 \\
$\quad$ Clorpirifós & 1,0 & & \\
a El tiempo de exposición al insecticida fue de 1 hora. Se evaluaron 300 mosquitos con cada \\
insecticida.
\end{tabular}

trato alfa naftilacetato (6) - es un elemento más que permite relacionar la resistencia encontrada al temefós con el mecanismo de las esterasas. Se ha demostrado en diversos países que la resistencia de $A$. aegypti al temefós está relacionada con la esterasa A4 (8, 9, 21-22).

La mayor actividad de las esterasas encontrada en las muestras estudiadas y la detección de la esterasa A4 constituyen indicadores de la resistencia al temefós en la localidad salvadoreña estudiada. En investigaciones realizadas por Mazzari en dos cepas de A. aegypti colectadas en los estados de Coro y Maracay, Venezuela, se observaron niveles moderados de resistencia al temefós, lo que se asoció con la presencia de una banda electroforética correspondiente a una es- terasa con movilidad relativa de 0,61 en 91\% de los mosquitos analizados. Esta esterasa, clasificada como A5, no presentó niveles altos de actividad en la cepa susceptible (26). En trabajos llevados a cabo en Brasil se asoció la resistencia a los insecticidas con la aparición de nuevas bandas de esterasas y la variación de su frecuencia entre los años 2000 y $2005(27,28)$.

En el presente trabajo se encontró una moderada resistencia del mosquito adulto a los piretroides deltametrina y cipermetrina y una susceptibilidad total a la lambdacialotrina y al insecticida organofosforado clorpirifós, según la clasificación recomendada por la OMS en 1981 (4), por lo que la lambdacialotrina y el clorpirifós son buenos candidatos para emplear en las estrategias de control de este vector. Se debe señalar que otros países de la Región han utilizado con éxito estos insecticidas en sus programas de control de $A$. aegypti. Por ejemplo, en Costa Rica se logró una letalidad de $97 \%$ a $100 \%$ con la lambdacialotrina, tanto en tratamientos de ultra bajo volumen, como en tratamientos térmicos (29). La estrategia utilizada en Cuba con la cipermetrina en esquemas de rotación con clorpirifós, tanto durante la epidemia de 1997 como en la de 2001-2002, puede haber evitado la aparición de la resistencia a la cipermetrina, lo que permitió reducir los índices de infestación con este vector en el año 2006 (30) solamente con este piretroide.

Los resultados obtenidos demuestran que la resistencia al temefós, sustentada por el mecanismo de las esterasas - específicamente de la esterasa A4-, podría reducir la eficacia del control químico del mosquito $A$. aegypti en la zona estudiada de El Salvador. No obstante, los insecticidas clorpirifós y la lambdacialotrina resultaron buenos candidatos alternativos a utilizar en las intervenciones de control de este vector.

Agradecimientos. Se agradece a la Organización Panamericana de la Salud por el apoyo financiero brindado a esta investigación.

\section{REFERENCIAS}

1. Vargas MJ. El dengue una enfermedad endémica en El Salvador. Nuevo Enfoque. 2007;II(0). Hallado en http://www.libros. com.sv/edicion0A/eldengue.html. Acceso el 21 de agosto de 2009.

2. Zaim M, Guillet P. Strategy development and monitoring for parasitic diseases and vector control. Trends Parasitol. 2002;18:161-3.

3. Instituto de Salud, Ambiente y Trabajo. Diagnóstico situacional del uso de DDT y el control de la malaria. Informe regional para México y Centroamérica. México, D.F.: Instituto de Salud, Ambiente y Trabajo; sin año. Hallado en http://www.cec.org/files/PDF/ pollutants/InfRegDDTb_ES_EN.pdf. Acceso el 3 de agosto de 2009.

4. World Health Organization. Instructions for determining the susceptibility or resistance of mosquito larvae to insecticides. Geneva: WHO; 1981. (WHO/VBC/81.80).

5. Wirth MC, Georghiou GP. Selection and characterization of temephos resistance in a population of Aedes aegypti from Tortola, British Virgin Islands. J Am Mosq Control Assoc. 1999;15:315-20.
6. Rodríguez MM, Bisset JA, Mila L, Calvo E, Díaz C, Soca LA. Niveles de resistencia a insecticidas y sus mecanismos en una cepa de Aedes aegypti de Santiago de Cuba. Rev Cub Med Trop. 1999;51:93-8.

7. Rodríguez MM, Bisset JA, Milá L, Molina D, Calvo E, Díaz C, et al. Levels of malathion resistance in Aedes aegypti and Culex quinquefasciatus as a result of use in Aedes aegypti control programs. J Am Mosq Control Assoc. 2000; 16:324-30.

8. Rodríguez MM, Bisset JA, Molina DF, Lauzan L, Soca A. Detection of resistance mechanisms in Aedes aegypti from Cuba and Venezuela. J Med Entomol. 2001;38:623-8.

9. Bisset JA, Rodríguez MM, Fernández D, Pérez O. Estado de la resistencia a insecticidas y mecanismos de resistencia en larvas del Municipio Playa, colectadas durante la etapa intensiva contra el Aedes aegypti en Ciudad de la Habana, 2001-2002. Rev Cub Med Trop. 2004;56:61-6.

10. World Health Organization. Pesticides and their application. 6th ed. Geneva: WHO; 2006. (WHO/CDS/WHOPES/GCDPP/2006.1.)
11. Raymond M. Present d'un programme $d^{\prime}$ analyse log-probit pour microordinateur. Cahiers Orstrom Sér Ent Med Parasitol. 1985;23:117-21.

12. Booth JE, Boyland E, Sims P. An enzyme from the rat liver catalyzing conjugation with glutathione. Biochem J. 1961;79:516-23.

13. Hemingway J, Boddington RG, Harris J. Mechanisms of insecticide resistance in Aedes aegypti (L.) (Diptera: Culicidae) from Puerto Rico. Bull Entomol Res. 1989;79:123-30.

14. World Health Organization. Vector resistance to pesticides. Fifteenth report of the WHO Expert Committee on Vector Biology and Control. Geneva: WHO; 1992. (WHO Technical Report Series No. 818.)

15. Braga IA, Da-Cunha MO, da Silva RC, Galardo AK, Soares Sda S, Braga IA, et al. Resistance of Aedes aegypti to organophosphates in several municipalities in the state of Rio de Janeiro and Espiritu Santo, Brazil. Am J Trop Med Hyg. 2003;68:329-33.

16. Braga IA, Lima JB, da Silva S, Valle D. Aedes aegypti resistance to temephos during 2001 in several municipalities in the state of Rio de 
Janeiro, Sergipe, and Alagoas, Brazil. Mem Inst Oswaldo Cruz. 2004;99:199-203.

17. Braga IA, Mello CB, Montella IR, Lima JB, Martins A, Medeiros PF, et al. Effectiveness of methroprene, and insect growth regulator, against temephos resistant Aedes aegypti populations from different Brazilian localities, under laboratory conditions. J Med Entomol. 2005;42:830-7.

18. Lima EP, de Oliveira Filho AM, de Oliveira Lima JW, Ramos Junior AN, de Góes Cavalcanti LP, Pontes RJ. Aedes aegypti resistance to temephos of Ceará State. Rev Soc Bras Med Trop. 2006;39:259-63.

19. Jirakanjanakit N, Saentharatip S, Rongnoparut P, Duchon S, Bellec C, Yoksan S. Trend of temephos resistance in Aedes (Stegomya) mosquitoes in Thailand during 2003-2005. Environ Entomol. 2007;36:506-11.

20. Vaughan A, Chadee DD, Ffrench-Constant, R. Biochemical monitoring of organophosphorus and carbamate insecticide resistance in Aedes aegypti mosquitoes from Trinidad. Med Vet Entomol. 1998;12:318-21.

21. Rodríguez MM, Bisset JA, Ruiz M, Soca A. Cross-resistance to pyrethroid and organophosphorus insecticides induced by selection with temephos in Aedes aegypti (Diptera: Culicidae) from Cuba. J Med Entomol. 2002;39:882-8.

22. Rodríguez MM, Bisset JA, Fernández D. Levels of insecticide resistance and resistance mechanisms in Aedes aegypti (Diptera: Culicidae) from some Latin American countries. J Am Mosq Control Assoc. 2007;24:420-9.

23. Bisset JA, Rodríguez MM, Fernández D. Resistencia a insecticidas y mecanismos de resistencia en Aedes aegypti (Diptera: Culicidae) de dos provincias del Perú. Rev Cub Med Trop. 2007;58:210-6.

24. Saelim V, Brogdon WG, Rojanapremsuk J, Suvannadaba S, Pandii W, Jones JW, et al. Bottle and biochemical assays on temephos resistance in Aedes aegypti in Thailand. Southeast Asian J Trop Med Public Health. 2005;36: 417-25.

25. Boyer S, David JP, Rev D, Lemperiere G, Ravanel P. Response of Aedes aegypti (Diptera: Culicidae) larvae to three xenobiotic exposures: larval tolerance and detoxifying activities. Environ Toxicol Chem. 2006;25:470-6.

26. Mazarri MB. Insecticide resistance in two field populations of Aedes aegypti (L.) from Venezuela [thesis of master degree]. California: University of Riverside; 1994.
27. Souza-Polezzi RC, Bicudo HE. Genetic variation along time in a Brazilian population of Aedes aegypti (Diptera: Culicidae), detected by change in the esterase patterns. Genetica. 2005;125:43-53.

28. Perich MJ, Rocha NO, Castro AL, Alfaro AW Platt KB, Solano T, et al. Evaluation of the efficacy of lambda-cyhalothrin applied by three spray application methods for emergency control of Aedes aegypti in Costa Rica. J Am Mosq Control Assoc. 2003;19:58-62.

29. Chávez J, Vargas J, Vargas F. Resistencia a deltametrina en dos poblaciones de Aedes aegypti (Diptera: Culicidae) del Perú. Rev Perú Biol. 2005;12:161-4.

30. Montada D, Zaldívar J, Sánchez F, Figueredo D, Suárez S, Leyva M. Eficacia de los tratamientos intradomiciliarios con los insecticidas cipermetrina, lambdacialotrina y clorpirifos en una cepa de Aedes aegypti. Rev Cub Med Trop. 2006;58:130-5.

Manuscrito recibido el 20 de junio de 2008. Aceptado para publicación, tras revisión, el 8 de febrero de 2009.

ABSTRACT Objectives. To assess the level of insecticide susceptibility of a certain Aedes aegypti strain found in El Salvador and to explain the mechanisms for its resistance to temephos. Methods. An A. aegypti strain from the municipality of Soyapango, Department of

\section{Assessing the insecticide resistance of an Aedes aegypti strain in El Salvador}

San Salvador, El Salvador, was studied. Bioassays were used to determine the susceptibility of the larvae to the organophosphate insecticide temephos and to three pyrethroids (deltamethrin, lambda-cyhalothrin, and cypermethrin); and of adults to an organophosphate insecticide (chlorpyrifos). The resistance factor $\left(\mathrm{RF}_{50}\right)$ with determined with respect to a reference susceptible strain (Rockefeller). The mechanism of temephos resistance was determined through the use of synergistic substances, biochemical assays for enzymatic activity, and polyacrylamide gel zymograms.

Results. The larvae of the strain studied proved highly resistant to temephos $\left(\mathrm{RF}_{50}=24.16\right)$. Of the enzyme samples analyzed, only the esterase A4 was linked to the mechanism of temephos resistance. The adult mosquitoes were susceptible to lambda-cyhalothrin and chlorpyrifos; and resistance to deltamethrin and cypermethrin fell into the category needing further verification.

Conclusions. Temephos resistance could reduce the efficiency of chemical control of the $A$. aegypti mosquito in El Salvador study area. Chlorpyrifos, lambda-cyhalothrin, and cypermethrin are good alternative insecticides for use in new efforts to control this vector.

Key words Aedes; temefos; insecticide resistance; vector control; El Salvador. 